\title{
Frequency Stability of the Cooler Pulsating White Dwarfs
}

\author{
D. J. Sullivan \\ School of Chemical \& Physical Sciences, Victoria University of \\ Wellington, P.O. Box 600, Wellington, New Zealand
}

\begin{abstract}
Although the frequencies of the pulsating white dwarfs are extremely stable, the evolutionary cooling of these geometrically constrained objects should lead to an observable period change over a sufficiently long time base. Direct measurement of a period change rate for the cooler white dwarfs should provide fundamental data on the core composition of these stellar remnants, and hence their cooling time scales. Progress on attempts to measure the period change rates of two white dwarfs is reported.
\end{abstract}

\section{Introduction}

Stellar evolution theory predicts that most stars will end up as white dwarfs. Studying the composition of these relatively "simple" stellar corpses should yield information about the prior evolutionary phases that may otherwise remain disguised due to their greater complexity. The chemical composition of white dwarf cores has a direct impact on their cooling time scales. Although the composition of a white dwarf core is usually assumed to be a ${ }^{12} \mathrm{C} /{ }^{16} \mathrm{O}$ mixture, this is based almost totally on the uncertain cross section values for the ${ }^{12} \mathrm{C}(\alpha, \gamma){ }^{16} \mathrm{O}$ reaction at particle energies corresponding to red giant cores (e.g. Wallerstein et al. 1997). Precise knowledge of white dwarf cooling time scales also has an important application outside the field of stellar structure studies: the coolest white dwarfs can be used to determine the age of the galactic disk.

Fortunately, nature has provided us with a way to probe the interiors of white dwarfs: some of them are g-mode pulsators, and knowledge of the pulsation modes combined with the tools of asteroseismology can yield fundamental structural information. Asteroseismology is most effective when armed with many detected pulsation modes, but pulsators with few modes are more likely to provide data on pulsation period stability within an evolutionary cooling environment. This, in turn, can be used to infer core chemical composition information.

\section{The Hydrogen Atmosphere White Dwarf L 19-2}

The pulsation properties of the southern DAV white dwarf L 19-2 ( $T_{\text {eff }} \sim 12,000$ $\mathrm{K}$ ) have been extensively studied by O'Donoghue \& Warner (1987) using photometric observations from a single site over a time base approaching $10 \mathrm{yr}$. Due to the sparseness of the number of detected pulsation modes in this work, the 
asteroseismological probe of the object's structure did not achieve its full potential. However, the existence of a dominant mode (period about $192 \mathrm{~s}$ ) provided the opportunity of monitoring the stability of a pulsation mode of this star, using data obtained from a single site. Within the measurement uncertainty of the nearly ten year dataset, the 192-s period was found to be stable by O'Donoghue \& Warner.

The benchmark for the measurement of period stability of white dwarf pulsation modes has been set by the work of Kepler and collaborators (e.g. Kepler, Nather, \& Metcalfe 1998). High speed photometry on the DAV object G117-B15A over a time base exceeding 25 yr has established that the dominant 215-s pulsation mode observed in this star has a stability that is rapidly approaching the theoretical limit of $d \Pi / d t=\dot{\Pi} \sim 10^{-15} \mathrm{~s} \mathrm{~s}^{-1}$.

With the aim of searching for more pulsation modes, and continuing the monitoring of pulsation stability, a Whole Earth Telescope (WET) run was carried out on L 19-2 in 1995. The WET multi-site high-speed photometry dataset has been supplemented by single-site data obtained by the author at $\mathrm{Mt}$ John Observatory (NZ) in the period 1994 to 1999. Some preliminary results of this work have already been published (Sullivan 1998), but a more complete description of the overall dataset and its analysis is currently in preparation (Sullivan et al. 2000a). It is anticipated that the period stability analysis will provide competition for the analysis of G117-B15A data by Kepler et al.

\section{The Helium Atmosphere White Dwarf EC 20058-5234}

The object EC 20058-5234 is a southern Helium atmosphere white dwarf $\left(\mathrm{T}_{\text {eff }} \sim\right.$ $25,000 \mathrm{~K}$ ) that was discovered as part of the Edinburgh-Cape survey of faint blue objects. Its classification and pulsation properties were first reported by Koen et al. (1995). As this object looked a promising candidate for asteroseismological analysis, a WET run was undertaken in July 1997. The superior multi-site WET dataset revealed a number of new pulsation modes and also demonstrated that the previously detected 256-s mode was sufficiently isolated in frequency space that it could be resolved with single-site photometry. Theoretical models of the hotter DB objects indicate that evolutionary cooling should have a greater impact on the pulsation modes, in comparison with their cooler DA cousins. The author has been monitoring EC 20058-5234 at Mt John Observatory, since the 1997 WET run. A report on this work is in preparation (Sullivan et al. 2000b).

\section{References}

Kepler, S. O., Nather, R. E., \& Metcalfe, T. S. 1998, Baltic Astronomy, 7, 175

Koen, C., O'Donoghue, D., Stobie, R. S., et al. 1995, MNRAS, 277, 913

O'Donoghue D. \& Warner B. 1987, MNRAS, 228, 949

Sullivan, D. J. 1998, Baltic Astronomy, 7, 159

Sullivan, D. J. et al. (the WET collaboration) 2000a, (in preparation)

Sullivan, D. J. et al. (the WET collaboration) 2000b, (in preparation)

Wallerstein, G., Iben, I., Parker, P., et al. 1997, Rev. Mod. Phys., 69, 995 